\title{
ArfGAPs; Depletion or overexpression?
}

\author{
Yoko Shiba* \\ Laboratory of Intracellular Traffic, Faculty of Science and Engineering, Iwate University, Japan
}

\begin{abstract}
Arf proteins are small GTP binding proteins that regulate intracellular traffic, actin remodeling and lipid metabolism. In the GTP-bound state, Arf is thought to be an active form that can bind to organelle membranes and many effectors. Arf in the GDP-bound state is thought to be an inactive form and released to cytosol. Arf GTPase Activating Proteins (ArfGAPs) hydrolyze Arf•GTP to Arf·GTP. Because GAPs "inactivate" small GTPases, the research of GAPs has often been done by overexpression of the GAPs looking at the biological consequences of the overexpression. However, decades of study about ArfGAP1 in COPI coated vesicle formation has led to a molecular model by which ArfGAP1 plays a role in fidelity of cargo sorting. This model implies that a deletion experiment would be appropriate to analyze the function of ArfGAP family proteins, rather than overexpression. Here, I briefly describe the role of ArfGAP1 in COPI coated vesicle formation, and discuss the biological consequences of depletion of ArfGAPs.
\end{abstract}

\section{Introduction}

In mammalian cells, intracellular traffic plays an important role in regulating cellular function. When intracellular traffic is impaired, intracellular proteins and lipids go to the wrong destinations, which causes organelle dysfunction that is often related to human disorders including cancer [1], neurodegenerative disorders [2], diabetes [1], and immune disorders [2]. Therefore, understanding the mechanism of intracellular traffic has primary importance for understanding human disorders, as well as biological functions of cells.

Secretory proteins, transmembrane proteins, and lysosomal enzymes are synthesized in the Endoplasmic Reticulum (ER), and then transported to the Golgi apparatus. In the Golgi, they are modified by glycosylation or other modifications, then sorted out into their destinations including the lysosomes, secretory granules, and plasma membrane. On the other hand, cells also internalize proteins from outside. These endocytosed proteins are transported to the early endosomes then sorted out to their destinations including the lysosomes where the proteins are digested by lysosomal enzymes. These transport processes are mediated by the transport vesicles that are often coated by coat proteins. There are three main classes of coat proteins, COPI, COPII, and clathrin. COPII coated vesicles mediate ER-Golgi transport, COPI coated vesicles mediate transport between Golgi stacks, or from the Golgi to the ER transport, and clathrin coated vesicles mediate post-Golgi traffic and endocytosis [3]. These coat proteins can bind to "cargo" proteins, which will be packaged into coated vesicles. Therefore, it has been thought that the binding between cargo and coat proteins mediates cargo sorting, the process which selects appropriate cargos into coated vesicles.

Arf/Sar family GTP-binding proteins are essential proteins to recruit coat proteins to organelle membranes [4,5]. Mammalian cells have 6 Arfs, Arf1 to Arf6. Human lacks Arf2, so there are 5 Arfs in human. Sar1 is the only protein in this family. Arf in its GTP-binding state is primarily considered to be an active form and binds to the organelle membranes by its myristoylated $\mathrm{N}$-terminal region [5], as well as a variety of Arf effectors including coat proteins [5,6]. Arf in its GDP-binding state is an inactive form and is present in cytosol. GTP and GDP binding states on Arf are regulated by Arf guanine nucleotide exchange factors (ArfGEFs) and Arf GTPase Activating Proteins (ArfGAPs) (Figure 1). ArfGEFs mediate the conversion of Arf•GDP to Arf•GTP [7], while ArfGAPs hydrolyze Arf•GTP into Arf•GDP and release Arf from the membrane to the cytosol [8]. In the traditional model, ArfGAPs terminate Arf signaling, therefore ArfGAPs have been thought to inhibit vesicle formation. However, decades of research have led to the idea that ArfGAPs are the key factors that actually determine if the interaction between coat its specific cargo proteins is taking place, which is the critical step for cargo sorting before the completion of vesicle formation. Because ArfGAPs had been thought to be negative regulators of Arf, overexpression of ArfGAPs might had been thought to be the appropriate approach to inhibit Arf and investigate the phenotype of inhibition of transport. However, the decades of research have shown that ArfGAP1 play a role in fidelity of cargo sorting, and that implicates the downregulation of ArfGAPs as an appropriate approach to investigate the biological function of ArfGAPs.

\section{Arf1 and ArfGAP1 play a role in "fidelity" of cargo sorting in COPI coated vesicle formation}

How ArfGAPs are involved in COPI vesicle formation has been the subject of dispute [9-11]. Coatomer is the coat protein used to form COPI coated vesicles at the Golgi apparatus, and is recruited to the Golgi membrane by Arf•GTP. In the traditional model, coatomer binds to cargo, then polymerizes into COPI to deform membrane to produce COPI-coated vesicles [12]. In this model, Arf•GTP remains in the COPI vesicles, which predicts that the hydrolysis of Arf•GTP occurs after COPI coated vesicles are formed to release Arf together with coatomer from the vesicles. In this model, ArfGAPs are predicted

Correspondence to: Yoko Shiba, Laboratory of Intracellular Traffic, Faculty of Science and Engineering, Iwate University, 4-3-5, Ueda, Morioka, 020-8551, Japan, Tel: (+81)019-621-6311; E-mail: shibay@iwate-u.ac.jp

Key words: ADP-ribosylation factor (ARF), Arf GTPase-activating protein (ArfGAP), small GTPase, COPI, Clathrin, sorting

Received: November 22, 2017; Accepted: December 22, 2017; Published: December 26, 2017 


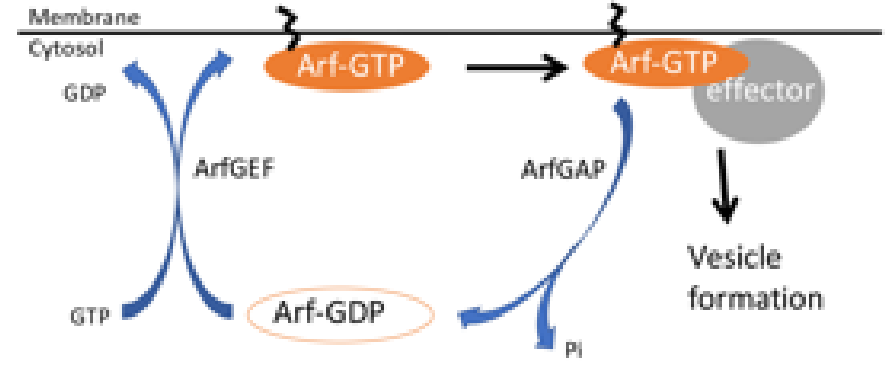

Figure 1. Molecular switch model of regulation of Arf. Arf.GTP is converted by Arf guanine nucleotide exchange factors (ArfGEFs), and Arf•GTP is hydrolyzed by Arf GTPase Activating Proteins (ArfGAPs). In this model, ArfGAPs play a role in deactivating Arf•GTP.

to be involved in uncoating. However, there have been reports that the hydrolysis of Arf•GTP is actually required for cargo incorporation into COPI coated vesicles [13-16]. By adding GTP区S, the non-hydrolyzable analogue of GTP, or by injecting an Arf1Q71L mutant that cannot hydrolyze GTP, the cargo incorporation into COPI vesicles was inhibited. As cargo sorting is thought to occur before the completion of vesicle formation, these data predict that GTP hydrolysis of Arf is required for cargo sorting before COPI coated vesicles are formed.

In this review, I will not describe the details of how the molecular model of COPI coated vesicle formation has been developed. Instead, readers can find it elsewhere [17]. I just introduce the model that I have proposed for ArfGAP1 (Figure 2); coatomer is recruited to the Golgi membrane by Arf.GTP and COPI binds to its cargo. In the presence of COPI and cargo, GAP activity of ArfGAP1 increases and hydrolyzes Arf•GTP [18]. Arf•GDP is released from the membrane and ArfGAP1 promotes polymerization of COPI to produce COPI coated vesicles [19]. In this model, GTP hydrolysis is required for determining if the interaction between COPI and its specific cargo is taking place (Figure 2 step 4 and 5). A GTP hydrolysis is the prerequisite for uncoating, but in contrast to the traditional model where the GTP hydrolysis takes place in the uncoating step, in our model, it takes place at the step in which cargo binds to coatomer. This model predicts that COPI remains after GTP hydrolysis on Arf. There are experimental data that the membrane dissociation rate of Arf1 is more rapid than that of coatomer in living cells [20], indicating that coatomer remains on the membrane after GTP hydrolysis on Arf1. Furthermore, recent studies involving structural analyses have suggested that the binding site of \-COP to Arfl•GTP might be transmitted to KDEL cargo receptor after GTP hydrolysis on Arf1 [21]. These studies support the model by which GTP hydrolysis on Arf is related to cargo sorting.

\section{Arfs function as sensors such as EF-Tu, rather than mo- lecular switches such as Ras family proteins}

Our model implies that Arf1 and ArfGAP1 function more like sensors such as EF-Tu, a bacterial translational GTPase that controls protein translation on ribosome, rather than as molecular switches such as Ras proteins $[22,23]$. In the case of EF-Tu, the correct pairing between mRNA-codon and tRNA-anticodon leads to the structural change of the ribosome to activate GTP hydrolysis on EF-Tu. Then EF-Tu dissociates from ribosome and further structural changes are anticipated for accommodation of aminoacyl tRNA into the peptidyl transferase center. GTP hydrolysis on EF-Tu is required for fidelity of codon-anticodon pairing, rather than required for terminating signals of GTP-bound EF-Tu. The role of Arf1/ArfGAP1 in COPI coated vesicle formation could be equivalent to EF-Tu, which raises many questions; Does coatomer play a role with ArfGAP1 as a GAP for Arf1, as an analogy to the ribosome as a GAP for EF-Tu? If so, does cargo induce structural changes of coatomer to activate GAP activity of ArfGAP1? What are the effects on coatomer after GTP hydrolysis on Arf1 with ArfGAP1? The answers to these questions remain elusive. A large body of biochemical and structural analyses will be required in the future.

\section{COPII and clathrin/AP-1 could also use small GTP binding proteins as sensors}

I described the hypothesis in which Arf1/ArfGAP1 plays a role in fidelity of cargo sorting in COPI coated vesicle formation. Can it also apply to other transport pathways? Reports concerning COPII and clathrin/AP-1 coated vesicles are in line with this hypothesis. Forster et al. analyzed the membrane turnover rates of Sarlp, a GTP binding protein required for COPII recruitment to ER membrane, and Sec23p/ Sec24p, the subunits of COPII coat and the GAP for Sarlp, at the site of ER exit sites in living cells [24]. The turnover rate of Sarlp was more rapid than that of Sec23p/24p. Decreasing the amount of secretory cargo slowed down the turnover of Sarlp and accelerated that of Sec23/24p. They concluded that COPII complex remains on the ER membrane after GTP hydrolysis on Sarlp, and the presence of cargo retains more COPII than Sarlp on the ER membrane. The results are consistent with the idea that GTP hydrolysis on Sarlp takes place for cargo sorting before completion of COPII coated vesicle formation.

AP-1 is a clathrin adaptor complex recruited to the Golgi/ endosomal membranes by Arf•GTP. The biochemical analyses of Zhu et al. showed that more AP-1 is retained on the Golgi membrane devoid of its cargo after 1M Tris extraction [25]. As COPII turnover was more rapid when secretory cargo decreased, the results of AP-1 appear to be opposite to COPII. However, AP-1 was extracted by $1 \mathrm{M}$ Tris even in the presence of GTP, which usually does not occur in cells. They proposed that AP-1 has two states for binding to the Golgi membrane; The one state has a high affinity to the Golgi membrane, which cannot be extracted by $1 \mathrm{M}$ Tris. This state occurs in the presence of GTP区S. On the other hand, the other state has a low affinity and can be extracted by $1 \mathrm{M}$ Tris biochemically. However, in cells, this low affinity state of AP-1 should also be on the membrane in the presence of GTP. Depletion of AP-1 cargo retains AP-1 in the high affinity state to the Golgi membrane even in the presence of GTP. They suggested that the affinity state of AP-1 is changed from high to low by GTP hydrolysis in

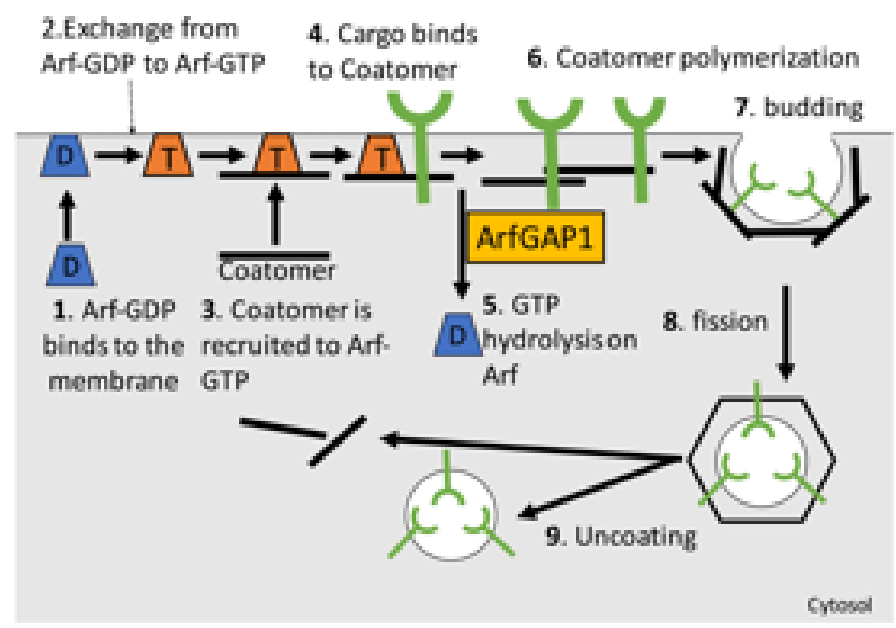

Figure 2. The model of COPI coated vesicle formation. ArfGAP1 mediates GTP hydrolysis on Arf when coatomer binds to its cargo. In this model, ArfGAP1 plays a role as a sensor if the coupling of coat and cargo is correct. 
the presence of cargo. In the absence of cargo, the change in the affinity state of AP-1 does not occur. Therefore AP-1 is retained in the high affinity binding state on the Golgi membrane. The low affinity state of AP-1 could be equivalent to the membrane-bound Sec23/24p after GTP hydrolysis of Sar1p. These reports imply that structural change of coat proteins could occur after GTP hydrolysis, and coat proteins are retained on organelle membrane for further vesicle formation, which suggests that the molecular model of COPI coated vesicle formation could apply to other transport pathways.

\section{ArfGAPs; depletion or overexpression?}

ArfGAPs family has 31 genes in human [26]. How can they be analyzed? In the traditional model, ArfGAPs function as terminators of Arf signaling, and one may think ArfGAPs should be overexpressed to hydrolyze more Arf•GTP to inhibit intracellular traffic and then analyze their phenotypes. Actually, in the case of Rab proteins, there are other small GTP binding proteins, and the research on RabGAPs has often been done by overexpression of RabGAPs [27-30]. But is this really the correct method for analyzing ArfGAPs?

Gcs1, yeast ArfGAP1 homologue, was found to be a suppressor of the loss of Arf function [31]. The authors suggested that the biological role of Gcs1 is a downstream effector of Arfs, rather than a downregulator. In mammalian cells, overexpression of ArfGAP1 produces more vesicles, rather than inhibiting transport [19]. The depletion of ACAP3, the ArfGAP for Arf6, resulted in the inhibition of neurite outgrowth [32]. Although Arf6•GTP levels increased by depletion of ACAP3, the shRNA of Arf6 also decreased neurite outgrowth. As the fast cycling mutant of Arf6, T157A rescued the phenotype of ACAP3 knockdown, but not GDP- and GTP- locked mutant of Arf6, Q67L and T44N, Miura et al. [33] concluded that the cycle of Arf6 is required for neurite outgrowth. Although the consequences of depletion of ArfGAPs in terms of Arf•GTP levels should be addressed carefully, accumulating evidence [33-40] shows that ArfGAPs are able to positively regulate biological processes.

siRNA screens of ArfGAPs, but not overexpression, have been done for analyzing intracellular traffic. The screen by 6 siRNAs of ArfGAPs determined AGAP2 as a positive regulator for transport of Shiga Toxin $\mathrm{B}$ subunit $(\mathrm{STxB})$ from the early endosomes to the Golgi apparatus [41]. The screen by 25 siRNAs of ArfGAPs determined ArfGAP3 to be required for Mannose 6-phosphate receptor (MPR) transport from the early endosomes to the late endosomes [42]. In this case, GAP activity of ArfGAP3 is found to be required for MPR transport, and a cathepsin D pulse-chase experiment confirmed the inhibition of MPR transport by ArfGAP3 depletion. One may still think that the depletion of ArfGAPs should increase Arf•GTP levels in cells, therefore intracellular traffic should be accelerated. Actually, I have often seen that the depletion of several different ArfGAPs resulted in the increase of the transport pathway of interest ([41] data not shown). These phenomena could be explained by the increase of Arf•GTP levels generally in cells. But is it specific to cargo? The simple increase of Arf•GTP levels could retain coat proteins such as AP-1 in a high affinity binding state to membranes. But is it possible to make vesicles? Let's consider the AP-1 pathway. If the particular ArfGAP that regulates the AP-1 pathway is depleted, AP-1 might not be transmitted from the high affinity binding state to the low affinity binding state, which may result in the inhibition of transport. AP-1 remaining on the membrane could be released by other ArfGAPs slowly that are not specific to AP-1 and its cargo, as there are 31 genes of ArfGAPs in human. These possibilities need to be addressed in each coat and ArfGAPs; however, the molecular model in which ArfGAP1 plays a role in fidelity of cargo sorting suggests that depletion experiments should be considered to be appropriate for analyses of other ArfGAPs, rather than overexpression.

Recently Shin JHH et al. reported that one of the RabGAPs, TBC1D23, binds to golgin-97/245 at the Golgi and links to the WASH complex on endosome-derived vesicles [43]. TBC1D23 lacks the arginine and glutamine residues essential for RabGAP activity. They used the CRISPR-Cas9 system to generate null mutants of TBC1D23 in HeLa cells. They suggested that TBC1D23 plays a role in the specificity of vesicle fusion to the target organelles. This work implies that depletion experiments should be generally considered to analyze the role of GAPs for small GTPases.

\section{Acknowledgement}

Thank you to Paul Langman, Ph.D. for assistance with the usage of Scientific English.

\section{References}

1. Wang C, Telpoukhovskaia MA, Bahr BA, Chen X, Gan L (2017) Endo-lysosomal dysfunction: a converging mechanism in neurodegenerative diseases. Curr Opin Neurobiol 48: 52-58. [Crossref]

2. Leto D, Saltiel AR (2012) Regulation of glucose transport by insulin: traffic control of GLUT4. Nat Rev Mol Cell Biol 13: 383-396. [Crossref]

3. Bonifacino JS, Glick BS (2004) The mechanisms of vesicle budding and fusion. Cell 116: 153-166. [Crossref]

4. Kahn RA, Cherfils J, Elias M, Lovering RC, Munro S, et al. (2006) Nomenclature for the human Arf family of GTP-binding proteins: ARF, ARL, and SAR proteins. $J$ Cell Biol 172: 645-650. [Crossref]

5. Kahn RA (2009) Toward a model for Arf GTPases as regulators of traffic at the Golgi. FEBS Lett 583: 3872-3879. [Crossref]

6. Nie Z, Hirsch DS, Randazzo PA (2003) Arf and its many interactors. Curr Opin Cell Biol 15: 396-404. [Crossref]

7. Donaldson JG, Jackson CL (2011) ARF family G proteins and their regulators: roles in membrane transport, development and disease. Nat Rev Mol Cell Biol 12: 362-375. [Crossref]

8. Shiba Y, Randazzo PA (2014) ArfGAPs: key regulators for receptor sorting. Receptors Clin Investig 1: e158. [Crossref]

9. Beck R, Brügger B, Wieland F (2011) GAPs in the context of COPI: Enzymes, coat components or both? Cell Logist 1: 52-54. [Crossref]

10. Kahn RA (2011) GAPs: Terminator versus effector functions and the role(s) of ArfGAP1 in vesicle biogenesis. Cell Logist 1: 49-51. [Crossref]

11. Hsu VW (2011) Role of ArfGAP1 in COPI vesicle biogenesis. Cell Logist 1: 55-56 [Crossref]

12. Nickel W, Wieland FT (1997) Biogenesis of COPI-coated transport vesicles. FEBS Lett 413: 395-400. [Crossref]

13. Nickel W, Malsam J, Gorgas K, Ravazzola M, Jenne N, et al. (1998) Uptake by COPIcoated vesicles of both anterograde and retrograde cargo is inhibited by GTPgammaS in vitro. J Cell Sci 111, 3081-3090. [Crossref]

14. Lanoix J, Ouwendijk J, Stark A, Szafer E, Cassel D, et al. (2001) Sorting of Golgi resident proteins into different subpopulations of COPI vesicles: a role for ArfGAP1.J Cell Biol 155: 1199-1212. [Crossref]

15. Pepperkok R, Whitney JA, Gomez M, Kreis TE (2000) COPI vesicles accumulating in the presence of a GTP restricted arf1 mutant are depleted of anterograde and retrograde cargo. J Cell Sci 113 (Pt 1): 135-144. [Crossref]

16. Yang JS, Lee SY, Gao M, Bourgoin S, Randazzo PA, et al. (2002) ARFGAP1 promotes the formation of COPI vesicles, suggesting function as a component of the coat. $J$ Cell Biol 159: 69-78. [Crossref]

17. Shiba Y, Randazzo PA (2012) ArfGAP1 function in COPI mediated membrane traffic currently debated models and comparison to other coat-binding ArfGAPs. Histol Histopathol 27: 1143-1153. [Crossref]

18. Luo R, Ha VL, Hayashi R, Randazzo PA (2009) Arf GAP2 is positively regulated by coatomer and cargo. Cell Signal 21: 1169-1179. [Crossref] 
19. Shiba Y, Luo R, Hinshaw JE, Szul T, Hayashi R, et al. (2011) ArfGAP1 promotes COPI vesicle formation by facilitating coatomer polymerization. Cell Logist 1: 139154. [Crossref]

20. Presley JF, Ward TH, Pfeifer AC, Siggia ED, Phair RD, et al. (2002) Dissection of COPI and Arf1 dynamics in vivo and role in Golgi membrane transport. Nature 417: 187-193. [Crossref]

21. Dodonova SO, Aderhold P, Kopp J, Ganeva I, Röhling S, et al. (2017) 9A structure of the COPI coat reveals that the Arfl GTPase occupies two contrasting molecular environments. Elife 6. [Crossref]

22. Carvalho AT, Szeler K, Vavitsas K, Åqvist J, Kamerlin SC3 (2015) Modeling the mechanisms of biological GTP hydrolysis. Arch Biochem Biophys 582: 80-90. [Crossref]

23. Voorhees RM, Ramakrishnan V (2013) Structural basis of the translational elongation cycle. Annu Rev Biochem 82: 203-236. [Crossref]

24. Forster R, Weiss M, Zimmermann T, Reynaud EG, Verissimo F, et al. (2006) Secretory cargo regulates the turnover of COPII subunits at single ER exit sites. Curr Biol 16: 173-179. [Crossref]

25. Zhu Y, Traub LM, Kornfeld S (1999) High-affinity binding of the AP-1 adaptor complex to trans-golgi network membranes devoid of mannose 6-phosphate receptors. Mol Biol Cell 10: 537-549. [Crossref]

26. Kahn RA, Bruford E, Inoue H, Logsdon JM, JNie Z, et al. (2008) Consensus nomenclature for the human ArfGAP domain-containing proteins. J Cell Biol 182: 1039-1044. [Crossref]

27. Fuchs E, Haas AK, Spooner RA, Yoshimura S, Lord JM, et al. (2007) Specific Rab GTPase-activating proteins define the Shiga toxin and epidermal growth factor uptake pathways. J Cell Biol 177: 1133-1143. [Crossref]

28. Yoshimura S, Gerondopoulos A, Linford A, Rigden DJ, Barr FA (2010) Family-wide characterization of the DENN domain Rab GDP-GTP exchange factors. $J$ Cell Biol 191: 367-381. [Crossref]

29. Longatti A, Lamb CA, Razi M, Yoshimura S, Barr F, et al. (2012) TBC1D14 regulates autophagosome formation via Rab11- and ULK1-positive recycling endosomes. $J$ Cell Biol 197, 659-675. [Crossref]

30. Biesemann A, Gorontzi A, Barr F, Gerke V (2017) Rab35 protein regulates evoked exocytosis of endothelial Weibel-Palade bodies. J Biol Chem 292: 11631-11640. [Crossref]
31. Zhang CJ, Cavenagh MM, Kahn RA (1998) A family of Arf effectors defined as suppressors of the loss of Arf function in the yeast Saccharomyces cerevisiae. $J$ Biol Chem 273: 19792-19796. [Crossref]

32. Miura Y, Hongu T, Yamauchi Y, Funakoshi Y, Katagiri N, et al. (2016) ACAP3 regulates neurite outgrowth through its GAP activity specific to Arf6 in mouse hippocampal neurons. Biochem J 473: 2591-2602. [Crossref]

33. Zhang S, Liu X, Li L, Yu R, He J, et al. (2017) The ArfGAP protein MoGlo3 regulate the development and pathogenicity of Magnaporthe oryzae. Environ Microbiol 19: 3982-3996. [Crossref]

34. Bist P, Kim SS, Pulloor NK, McCaffrey K, Nair SK, et al. (2017) ArfGAP DomainContaining Protein 2 (ADAP2) Integrates Upstream and Downstream Modules of RIG-I Signaling and Facilitates Type I Interferon Production. Mol Cell Biol 37. [Crossref]

35. Oku Y, Huganir RL (2013) AGAP3 and Arf6 regulate trafficking of AMPA receptors and synaptic plasticity. $J$ Neurosci 33: 12586-12598. [Crossref]

36. Dias M, Blanc C, Thazar-Poulot N, Ben Larbi S, Cosson P, et al. (2013) Dictyostelium ACAP-A is an ArfGAP involved in cytokinesis, cell migration and actin cytoskeleton dynamics. J Cell Sci 126: 756-766. [Crossref]

37. Ha VL, Bharti S, Inoue H, Vass WC, Campa F, et al. (2008) ASAP3 is a focal adhesionassociated Arf GAP that functions in cell migration and invasion. J Biol Chem 283 14915-14926. [Crossref]

38. Li J, Peters PJ, Bai M, Dai J, Bos E, et al. (2007) An ACAP1-containing clathrin coat complex for endocytic recycling. J Cell Biol 178: 453-464. [Crossref]

39. Onodera Y, Hashimoto S, Hashimoto A, Morishige M, Mazaki Y, et al. (2005) Expression of AMAP, an ArfGAP, provides novel targets to inhibit breast cancer invasive activities. EMBO J 24: 963-973. [Crossref]

40. Hashimoto S, Hashimoto A, Yamada A, Kojima C, Yamamoto H, et al. (2004) A novel mode of action of an ArfGAP, AMAP2/PAG3/Papa lpha, in Arf6 function. J Biol Chem 279: 37677-37684. [Crossref]

41. Shiba Y, Romer W, Mardones GA, Burgos PV, Lamaze C, et al. (2010) AGAP2 regulates retrograde transport between early endosomes and the TGN. J Cell Sci 123: 2381-2390. [Crossref]

42. Shiba, Y, Kametaka, S, Waguri, S, Presley, J. F, and Randazzo, P. A. (2013) ArfGAP3 regulates the transport of cation-independent mannose 6-phosphate receptor in the postGolgi compartment. Curr Biol 23: 1945-1951. [Crossref]

43. Shin JJH, Gillingham AK, Begum F, Chadwick J, Munro S1 (2017) TBC1D23 is a bridging factor for endosomal vesicle capture by golgins at the trans-Golgi. Nat Cell Biol 19: 1424-1432. [Crossref]

Copyright: (C2017 Shiba Y. This is an open-access article distributed under the terms of the Creative Commons Attribution License, which permits unrestricted use, distribution, and reproduction in any medium, provided the original author and source are credited. 